\title{
BMJ Global Health Spatial and spatio-temporal methods for mapping malaria risk: a systematic review
}

\author{
Julius Nyerere Odhiambo (D) , ${ }^{1}$ Chester Kalinda, ${ }^{1,2}$ Peter M Macharia (D) , ${ }^{3}$ \\ Robert W Snow, ${ }^{3,4}$ Benn Sartorius ${ }^{1,5}$
}

To cite: Odhiambo JN, Kalinda C, Macharia PM, et al. Spatial and spatiotemporal methods for mapping malaria risk: a systematic review. BMJ Global Health 2020;5:e002919. doi:10.1136/ bmjgh-2020-002919

Handling editor Alberto L Garcia-Basteiro

- Additional material is published online only. To view please visit the journal online (http://dx.doi.org/10.1136/ bmjgh-2020-002919).

Received 16 May 2020 Revised 23 August 2020 Accepted 24 August 2020

Check for updates

\section{(c) Author(s) (or their} employer(s)) 2020. Re-use permitted under CC BY-NC. No commercial re-use. See rights and permissions. Published by BMJ.

For numbered affiliations see end of article.

\section{Correspondence to} Julius Nyerere Odhiambo; nyererejulius7@gmail.com

\section{ABSTRACT}

Background Approaches in malaria risk mapping continue to advance in scope with the advent of geostatistical techniques spanning both the spatial and temporal domains. A substantive review of the merits of the methods and covariates used to map malaria risk has not been undertaken. Therefore, this review aimed to systematically retrieve, summarise methods and examine covariates that have been used for mapping malaria risk in sub-Saharan Africa (SSA).

Methods A systematic search of malaria risk mapping studies was conducted using PubMed, EBSCOhost, Web of Science and Scopus databases. The search was restricted to refereed studies published in English from January 1968 to April 2020. To ensure completeness, a manual search through the reference lists of selected studies was also undertaken. Two independent reviewers completed each of the review phases namely: identification of relevant studies based on the Preferred Reporting Items for Systematic Reviews and Meta-Analyses guidelines, data extraction and methodological quality assessment using a validated scoring criterion.

Results One hundred and seven studies met the inclusion criteria. The median quality score across studies was 12/16 (range: $7-16$ ). Approximately half (44\%) of the studies employed variable selection techniques prior to mapping with rainfall and temperature selected in over $50 \%$ of the studies. Malaria incidence $(47 \%)$ and prevalence $(35 \%)$ were the most commonly mapped outcomes, with Bayesian geostatistical models often (31\%) the preferred approach to risk mapping. Additionally, $29 \%$ of the studies employed various spatial clustering methods to explore the geographical variation of malaria patterns, with Kulldorf scan statistic being the most common. Model validation was specified in $53(50 \%)$ studies, with partitioning data into training and validation sets being the common approach.

Conclusions Our review highlights the methodological diversity prominent in malaria risk mapping across SSA. To ensure reproducibility and quality science, best practices and transparent approaches should be adopted when selecting the statistical framework and covariates for malaria risk mapping. Findings underscore the need to periodically assess methods and covariates used in malaria risk mapping; to accommodate changes in data availability, data quality and innovation in statistical methodology.

\section{Summary box}

\section{What is already known?}

- The disproportionate decline of malaria risk overtime and between/within countries in sub-Saharan Africa attributed to biological, environmental, social and demographic factors has triggered a renewed interest in its fine-scale epidemiology.

- Enhanced computational ability and availability of data of high quality and volume has enabled the quantification malaria risk burden in space and time leading to the proliferation of methods within a formal statistical framework.

- The complexity of spatio-temporal models has increased, making inferential and predictive processes difficult to undertake.

\section{What are the new findings?}

- The production of more granular estimates of malaria risk hinges on accessibility to and collection of timely data at finer resolutions.

- Variable selection should be objectively developed to contribute to the maximum predictive accuracy of the spatio-temporal model.

\section{What do the new findings imply?}

- Spatio-temporal approaches need to robustly quantify the sub-national burden of malaria risk, as an epidemiological prerequisite to intervention strategies.

- Investments in primary data collection at subnational scales, development and continuous application of robust modelling tools and approaches will be important for orienting malaria control and elimination efforts in the next decade.

- As the malaria landscape diversifies, new tools will be required to not only highlight changes locally, but also to provide evidence-based insights into factors driving the change.

\section{INTRODUCTION}

Global efforts to control and eliminate malaria are intrinsically linked to the Sustainable Development Goals. ${ }^{1}$ Specifically, the Global Technical Strategy (GTS) for Malaria (2016-2030) reiterates the need to reduce both malaria case incidence and mortality 
rates by up to $90 \%^{2}$ in high burden countries, mostly concentrated in sub-Saharan Africa (SSA), and eliminating malaria in at least 35 countries and preventing resurgence in malaria-free countries. ${ }^{2}$ However, in 2018, SSA had an estimated 213 million clinical episodes of malaria, caused mainly by Plasmodium falciparum parasite ${ }^{3}$ . To address this high burden, the GTS emphasises on the need to target interventions according to subnational disease risk stratification. ${ }^{2}$

The importance of malaria risk mapping in Africa can be traced back to the mid-1950s when malaria epidemiology formed a critical prelude to the design of interventions aimed at eliminating malaria. ${ }^{4} \mathrm{~A}$ resurgence in malaria cartography emerged in the $1990 \mathrm{~s},{ }^{4-6}$ coinciding with an era of intensive control and elimination activities. Over the last 20 years, national and subnational malaria risk maps have been developed in many endemic countries in SSA. ${ }^{48}$ This has led to a proliferation of methods and an increase in data quality and quantity-prompted by the demands for robust and reliable characterisation of malaria risk in space and time.

The science of malaria cartography has evolved from hand-drawn risk maps to contemporary digital maps due to the demand for computational solutions and methodologies. These are needed to produce accurate estimates at a high spatial and temporal resolution to facilitate monitoring elimination progress within and between countries in SSA. ${ }^{8}$ Modern mapping embracing novel statistical techniques at high spatial and temporal resolution are increasingly being used to inform public health policy. ${ }^{9}{ }^{10}$ Most recently, this has been aided by the availability of curated spatial databases, geographical information systems, enhanced computational capabilities and the advancement in spatial statistics. Standardised nationally representative survey initiatives, such as the geolocated Malaria Indicator Survey and the Demographic and Health Survey platforms, have availed geocoded malaria data with relevant covariates. ${ }^{11} 12$ This has enabled the characterisation of malaria risk at a high spatial resolution over which health policy is made.

Previous reviews have been conducted to; identify environmental risk factors of malaria transmission, ${ }^{11}{ }^{13}$ summarise methodological and computational power advancement. ${ }^{12}$ Despite the increase in the number of malaria risk mapping studies, there are no recent and comprehensive reviews of the changes in methodological frameworks and covariates used. Consequently, we aimed to identify and review malaria risk mapping studies, to assess analytical methods and covariates used in the last five decades.

\section{METHODS}

The protocol guiding this review has been previously published. ${ }^{14}$ Our results are reported according to the Preferred Reporting Items for Systematic Reviews and Meta-Analyses guidelines.$^{1516}$ A notable deviation from our protocol was limiting the review scope to SSA where the burden of malaria is highest and countries have broadly similar malaria vector and parasite ecologies and health system contexts, compared with low-income and middle-income countries. ${ }^{3}{ }^{17}$ A rigorous threephase process was undertaken to transparently identify and summarise spatio-temporal studies based on their methodical framework and covariates employed used in malaria risk mapping.

\section{Phase 1: Identification of relevant studies/keyword search.}

\section{Search terms and databases}

All studies published between 1 January 1968 and 30 April 2020 were systematically searched through four electronic databases (PubMed, Web of Science, EBSCOhost and Scopus) using search terms defined in online supplemental table 1. To improve the search strategy, thematically mined keywords were funnelled using Boolean operators and truncations before being employed across the selected electronic reference databases. The starting year (1968) corresponded to the year when the first global audit of malaria endemicity was undertaken. ${ }^{5}{ }^{12}$ Relevant studies were imported into Endnote, version X9 (Clarivate Analytics, Philadelphia, Pennsylvania, USA) (online supplemental table 1).

\section{Phase 2: Study selection}

Studies were screened independently by two authors JNO and CK for possible inclusion based on information provided in the title and abstract. Relevant studies based on the research questions were subsequently appraised on their eligibility for full-text review. The full-text review entailed the application of a more stringent inclusion/ exclusion criteria for selecting studies to be included for data extraction. Additional papers were identified by examining the reference lists of retrieved studies and by contacting the authors where necessary. Emerging discrepancies were resolved by consensus and by an independent arbitrator (BS). A comprehensive and pilot tested form was used for data extraction.

\section{Inclusion and exclusion criteria}

Peer-reviewed studies that employed spatial, temporal and spatio-temporal modelling techniques in malaria risk mapping in SSA were considered. A spatial model was defined as one that explicitly included a geographical index, while a temporal model included a time index. Studies using at least one visualisation or modelling technique (with or without covariates) for assessing the burden of malaria were included. Commentaries, expert reviews and/or reports that did not include original research were read, and only relevant studies cited included.

\section{Phase 3: Data extraction}

A standardised extraction form was used to independently extract the data by two reviewers (JNO and CK). The tool was first piloted and refined accordingly. Discordance between the reviewers with respect to the information 
extracted was resolved by consensus and by consulting with an independent arbitrator (BS). For each selected study, the following information was extracted (online supplemental table 2) namely:

i. Bibliographic information (Author, year, study setting and period, primary unit of analysis, spatial and temporal resolution).

ii. Study objective(s)

iii. Data aspects (data sources, malaria data, covariate type)

iv. Analytical method (modelling approach(es), assumptions, cluster detection techniques, statistical tests, diagnostic/validation checks).

v. Results and discussions (key findings, modelling gaps, recommendation(s)).

\section{Quality assessment}

A previously used 8-point scoring criteria ${ }^{18}$ was adapted and modified to assess the quality of the individual studies based on their aims and objectives, input data, model validity, results and conclusions (online supplemental table 3). Screening questions/criterion were used to guide the scoring process, with the score ranging from 0 (poor) to 2 (good) on each criterion. The overall quality level assigned to individual studies were summarised into four broad categories; very high $(>13)$, high (11-13), medium (8-10) and low $(<8)$ (online supplemental table 3).

\section{RESULTS}

\section{Literature search, data synthesis and quality assessment}

A total of 7189 studies were retrieved from the various databases with 170 studies fully screened after the title and abstract review. Ultimately 107 studies were included for

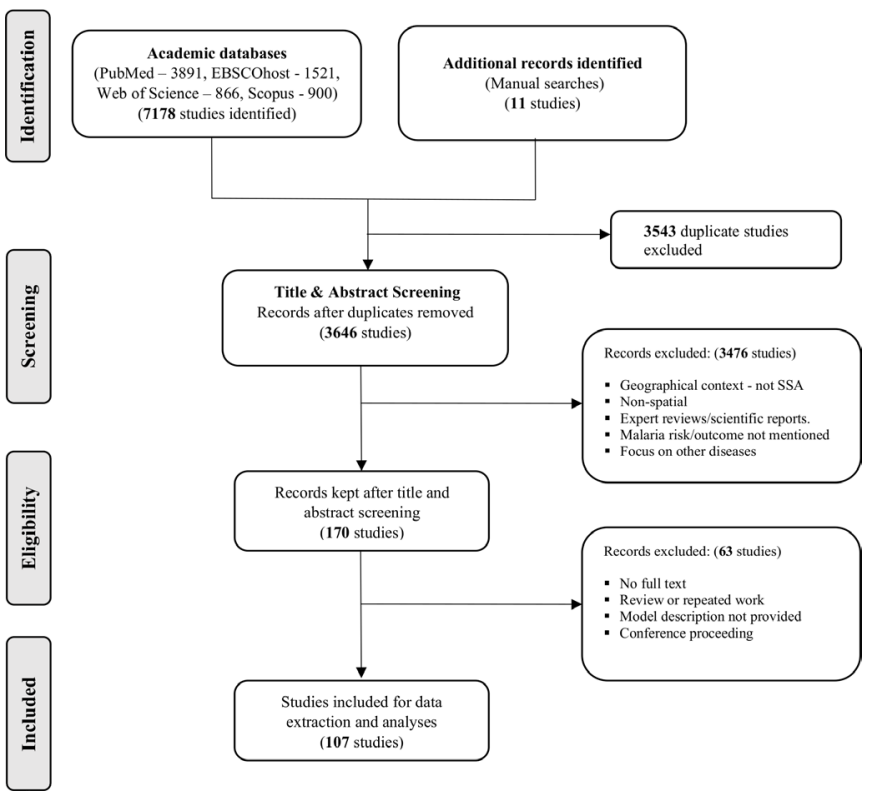

Figure 1 Study flow from literature search to data extraction and analyses. review and underwent quality assessment and synthesis (figure 1).

The distribution of studies by geographical scale and scope varied across SSA. Five (5\%) studies were continental in scale, $48(45 \%)$ studies were national and 52 (49\%) studies were subnational. Kenya (10 studies) and Tanzania (9 studies) had the highest number of publications included in the review (figure 2).

The longest study period spanned 115 years, ${ }^{19}$ while the shortest study period was 3 months. ${ }^{20}$ Fifty-eight $(54 \%)$ studies had an overall study period ranging between 3 months and 5 years, while $21(20 \%)$ studies had their study period ranging between 6 and 10 years and 28 (26\%) studies spanned more than 10 years. Overall, the number of publications increased over the review period (figure 3).

The median score was 12 out of 16 , with 16 representing the highest possible quality. The overall quality score of the reviewed studies ranged from 7 to 16 . Two studies were of low quality, 22 studies were of medium quality, 42 studies were of high quality and 41 studies were of very high quality (online supplemental table 3 ).

\section{Data sources, covariate selection and preprocessing}

From the review, global, continental, national and subnational databases/repositories provided a rich source of both malaria data and covariates used for modelling. These sources comprised of geographically referenced surveys used by 34 (32\%) studies, 20 (19\%) studies used population databases and $10(9 \%)$ studies used government records. Routinely collected data from the Health and Demographic Surveillance System were used in 16 $(15 \%)$ studies. Sources of climatic and environmental covariates consisted of ground station observations used by $17(16 \%)$ studies and remotely sensed satellite surrogates of climate, urbanisation and topography were employed by $49(46 \%)$ studies (table 1 ).

In this review, variable selection techniques were explicitly specified by $47(44 \%)$ studies. These techniques varied substantially; with the frequentist approach used in 14 $(13 \%)$ studies to assess the (uni and multi) variate association between malaria outcomes and its covariates being the most common. Significant covariates were included if their nominal $\mathrm{p}$ value was less than $0.001,{ }^{21-24} 0.05,{ }^{25-27}$ $0.1,{ }^{28} 0.15,{ }^{29}{ }^{30} 0.2 .^{31}{ }^{32}$ and $0.25 .^{33}$ The generalised linear models used by nine $(8 \%)$ studies identified the best covariate subset based on Wald's p value $\mathrm{e}^{203234-36}$ and the variance inflation factor. ${ }^{37}$ Additionally, six (6\%) studies used the total-sets analysis based on Bayesian information criterion (BIC) statistic to identify the optimal variable combination. Principal component analysis was employed by eight $(8 \%)$ studies to reduce dimensions and avoid collinearities in environmental factors, ${ }^{38} 39$ meteorological factors ${ }^{38} 40$ and household demographics. ${ }^{32} 33$ 41-43 The Bayesian stochastic search was used by three $(3 \%)$ studies to identify covariates with the highest inclusion probability. Other techniques employed included the least absolute shrinkage and selection operator (LASSO) 


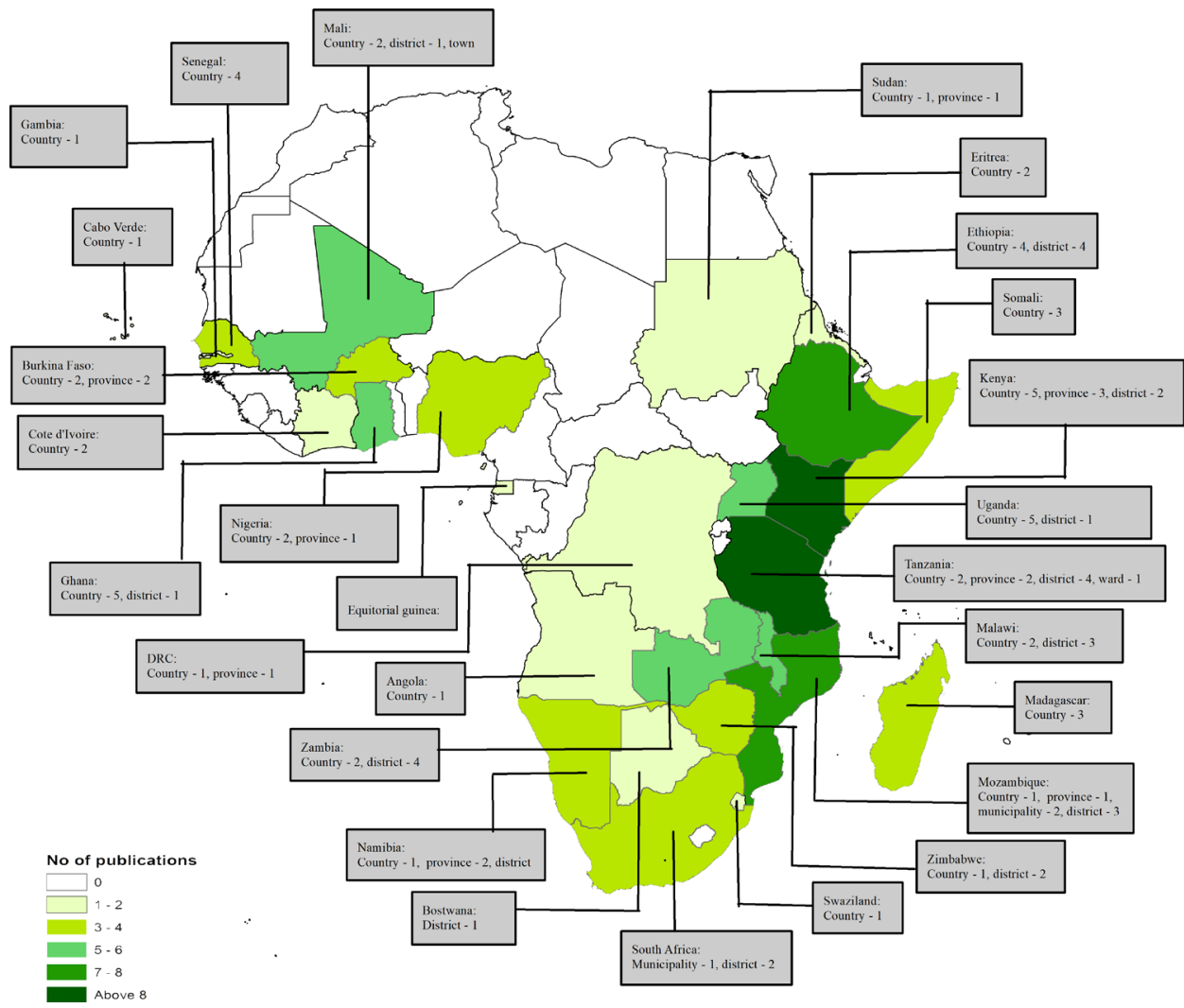

Figure 2 Geographical scale and scope of studies. Geographical scale (municipality, district, province/state, country) of studies is given in grey boxes. The studies covered 27 countries in sub-Saharan Africa with East Africa being the most represented subregion.

penalty, the Spike and slab and the Bayesian model averaging cumulatively used by five $(5 \%)$ studies. Two studies (2\%) reviewed covariates used in past studies to identify and adopt the best suite of covariates to be included in their model (table 2).

Data preprocessing procedures were employed in $37(35 \%)$ studies. The verification of geographical coordinates by either paper maps ${ }^{44}$ or global digital

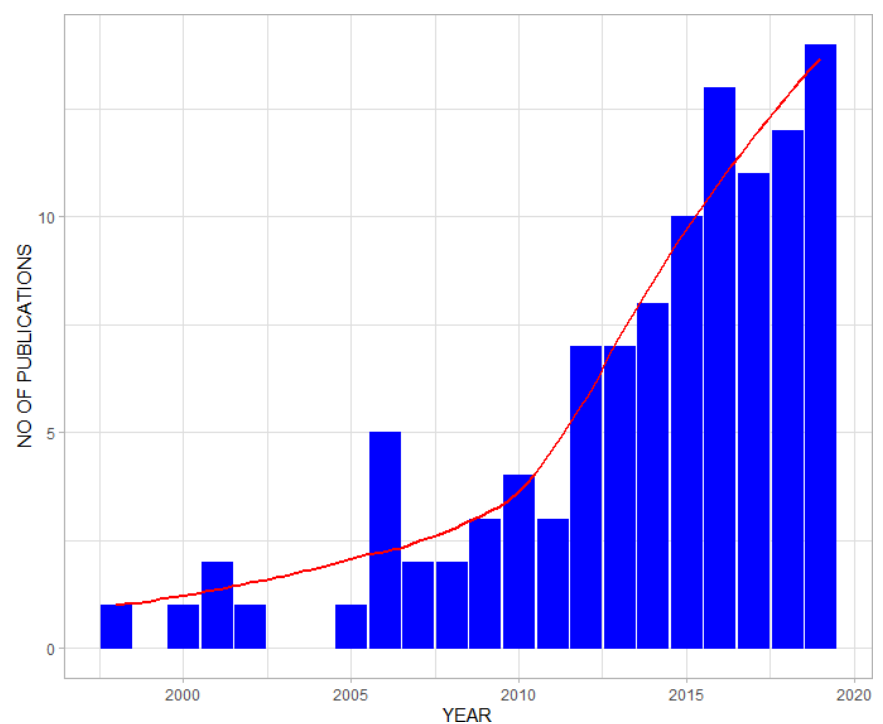

Figure 3 Bar - chart with a trend line (red) showing the total number of included studies. maps $83134364244-58$ used in $20(21 \%)$ studies was the most common procedure. Algorithms based on the catalytic conversion models ${ }^{8} 364648-505559-61$ were used in 10 (9\%) studies to generate age-adjusted malaria prevalence predictions, that is, age range of $2-10$ years. Continuous variables were standardised in seven $(7 \%)$ studies; by log transformation, ${ }^{62}$ centring on the mean ${ }^{4763-66}$ and zero. ${ }^{47}$ Four $(4 \%)$ studies excluded study regions with inconsistent datasets ${ }^{8196768}$ while two (2\%) studies used the average of its nearest values. ${ }^{69} 70$ Other approaches used included the multivariate stepwise regression, ${ }^{71}$ using data values extracted from previous surveys. ${ }^{32}$

\section{Modelling covariates}

The type and number of covariates included in malaria models varied across studies. Different categories encompassing climatic and environmental, sociodemographic and malaria intervention covariates were identified. The most common covariates in the environmental domain were rainfall and temperature used in $61(57 \%)$ and $59(55 \%)$ studies, respectively; while the most common sociodemographic covariates used in $12(11 \%)$ studies were population size and age. Malaria interventions (insecticide-treated bed nets, indoor residual spraying and artemisinin-based combined therapy) used in 32 $(30 \%)$ studies and transmission seasonality used in 28 (26\%) studies were also common. Detailed variations and adaptations covariates are presented in table 3 . 


\begin{tabular}{|c|c|c|c|}
\hline Type & Source & No & References \\
\hline \multirow[t]{8}{*}{$\begin{array}{l}\text { Global/continental } \\
\text { databases }\end{array}$} & $\begin{array}{l}\text { Malaria Transmission Intensity and } \\
\text { Mortality Burden across Africa }\end{array}$ & 1 & 88 \\
\hline & $\begin{array}{l}\text { Mapping Malaria Risk in Africa } \\
\text { databases }\end{array}$ & 9 & 293144456367739798 \\
\hline & World Pop/Afripop & 14 & $254146-485960727588$ 99-102 \\
\hline & $\begin{array}{l}\text { Food and Agriculture Organisation- } \\
\text { Food Security and Nutrition Analysis } \\
\text { Unit }\end{array}$ & 1 & 59 \\
\hline & $\begin{array}{l}\text { Global Rural and Urban Mapping } \\
\text { project }\end{array}$ & 2 & 4199 \\
\hline & $\begin{array}{l}\text { WHO database on malaria drug } \\
\text { resistance }\end{array}$ & 1 & 98 \\
\hline & Global Lakes and Wetlands Database & 3 & 344849 \\
\hline & $\begin{array}{l}\text { UN World Urbanisation prospects } \\
\text { database }\end{array}$ & 4 & 25495064 \\
\hline \multirow[t]{5}{*}{ National databases } & $\begin{array}{l}\text { Health and Demographic } \\
\text { Surveillance System }\end{array}$ & 16 & $2832384279100102-111$ \\
\hline & Census & 6 & 212268108112113 \\
\hline & National statistical agencies & 10 & $40516569107114-118$ \\
\hline & Demographic Health Survey & 7 & 232652616698101 \\
\hline & Malaria Indicator Survey & 12 & 37414349526066757699117119 \\
\hline \multirow[t]{4}{*}{ Subnational databases } & Cross-sectional surveys & 9 & 82046484953 120-122 \\
\hline & Cohort studies & 5 & 33395484 123-125 \\
\hline & Cluster surveys & 1 & 34 \\
\hline & $\begin{array}{l}\text { Entomological/parasitological } \\
\text { surveys }\end{array}$ & 5 & 77113120126127 \\
\hline \multirow[t]{14}{*}{ Remote sensing } & $\begin{array}{l}\text { Moderate Resolution Imaging } \\
\text { Spectroradiometer }\end{array}$ & 28 & $\begin{array}{l}2527293034373841485559-6165667275-7999100103104 \\
128-130\end{array}$ \\
\hline & Africa Data Dissemination Service & 8 & $2930657176-79$ \\
\hline & $\begin{array}{l}\text { United States Geological Survey- } \\
\text { Earth Resources Observation and } \\
\text { Science Centre }\end{array}$ & 8 & 2728303543767799 \\
\hline & Health Mapper & 8 & 272930416165687677 \\
\hline & Shuttle Radar Topographic Mission & 5 & 28607299129 \\
\hline & WorldClim-Global Climate database & 7 & $23343759-61100$ \\
\hline & Tropical Rainfall Measuring Mission & 3 & 104128130 \\
\hline & Early Warning System & 3 & 667288 \\
\hline & Climate Research Unit & 3 & 2371131 \\
\hline & $\begin{array}{l}\text { National Oceanic and Atmospheric } \\
\text { Administration }\end{array}$ & 2 & 109132 \\
\hline & Water Resources Institute & 1 & 63 \\
\hline & World Wildlife Fund & 1 & 37 \\
\hline & Africover & 1 & 34 \\
\hline & $\begin{array}{l}\text { Famine Early Warning Systems } \\
\text { Network Land Data Assimilation } \\
\text { System }\end{array}$ & 1 & ect \\
\hline Ground station data & Meteorological data & 17 & $21223538405469-7184103106113126$ 133-135 \\
\hline
\end{tabular}


Table 2 Analytical methods used in malaria risk mapping

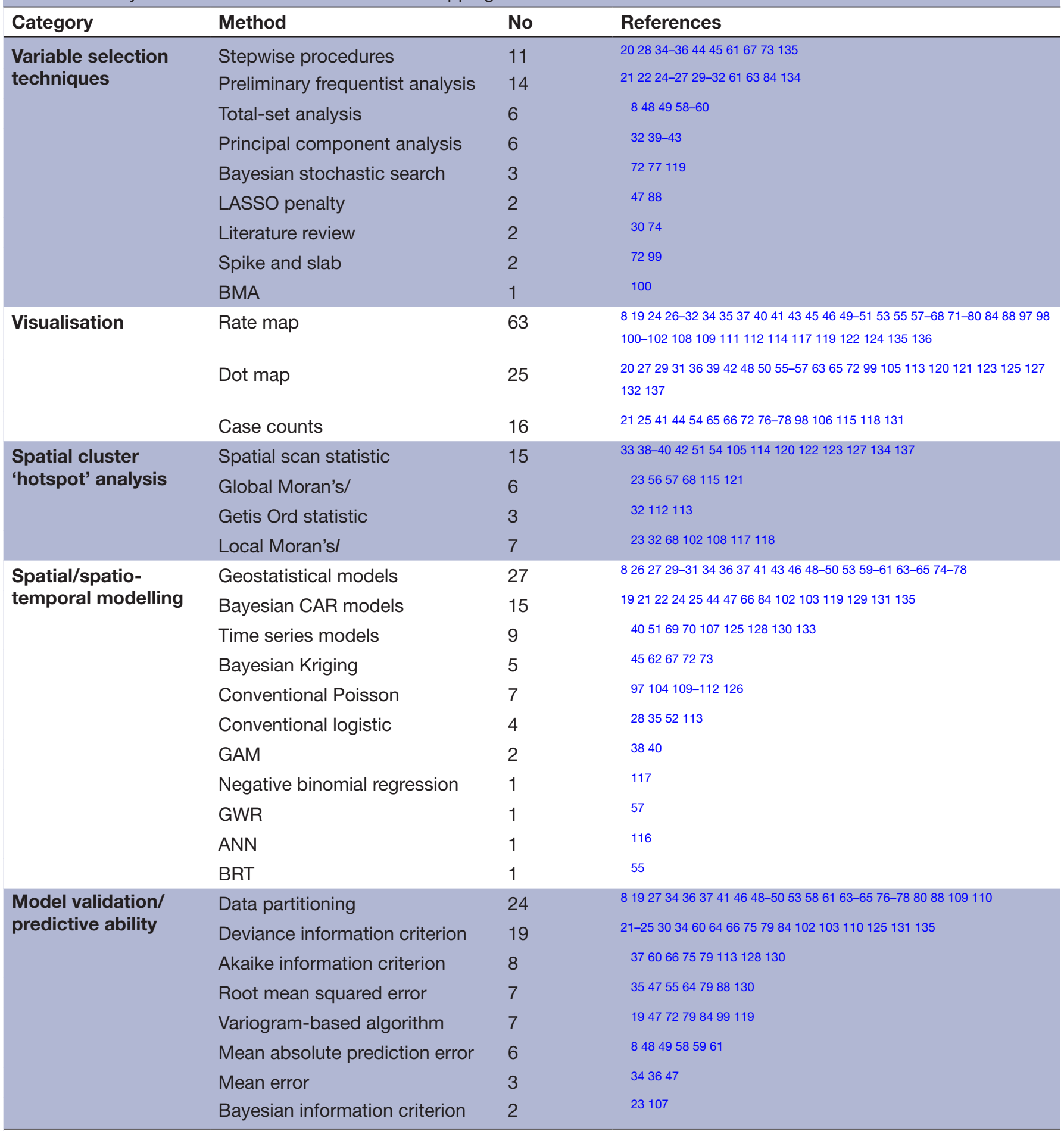

ANN, Artificial neural network; BMA, Bayesian model averaging; BRT, Boosted regression tree; CAR, Conditional autoregressive; GAM, General additive model; GWR, Geographically weighted regression; LASSO, Least absolute shrinkage and selection operator.

\section{Spatial, temporal and spatio-temporal methods}

A variety of spatial, temporal and spatio-temporal methods were employed to visualise malaria risk patterns, explore spatial clusters and model risk across space and time in SSA. Measurement of malaria burden varied across studies with the type of outcome informing the modelling framework. The most common malaria metric used in models, was incidence used in $50(47 \%)$ studies and prevalence used in 37 (35\%) studies. Table 3 presents a summary of the malaria outcomes that were considered in the papers included in the review.

In settings of low malaria transmission, local and global spatial cluster detection methods were used in 31 (29\%) studies to identify significant geographical variation in malaria risk patterns (table 2). These were the Kulldorf spatial scan statistic, Getis' Gi*(d) local statistic; 
Table 3 Covariates used in malaria risk mapping

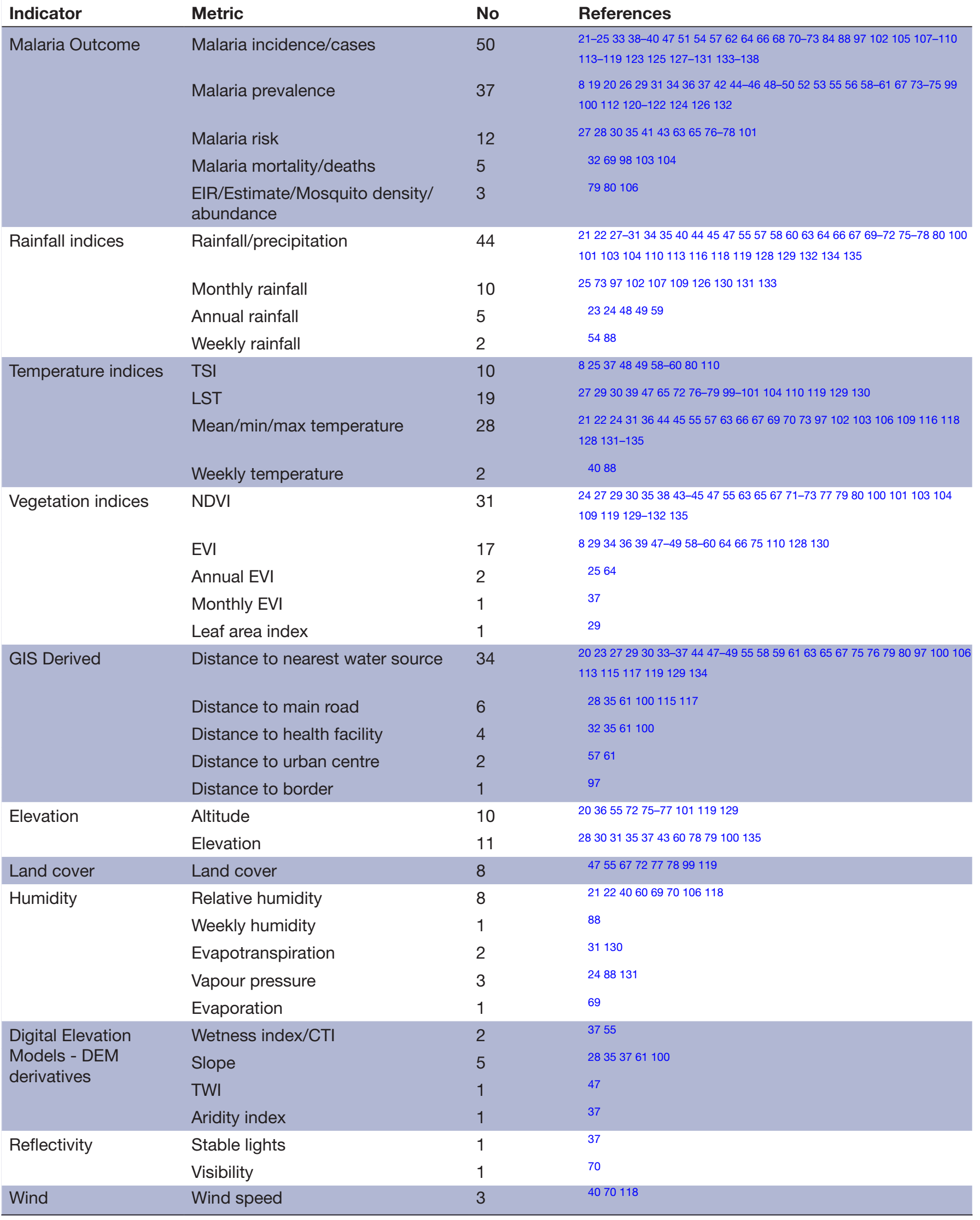

Continued 


\begin{tabular}{|c|c|c|c|}
\hline Indicator & Metric & No & References \\
\hline \multirow[t]{7}{*}{ Demographic factors } & SES & 9 & 2632424399100102108122 \\
\hline & Age & 12 & 2632424357758499100103106113 \\
\hline & Population density/size & 12 & 25374557677598100112115117135 \\
\hline & Urbanisation & 8 & $826364858-6072$ \\
\hline & Development & 1 & 113 \\
\hline & Wealth index/category & 4 & 7599119129 \\
\hline & Building/Housing material & 4 & 4257100123 \\
\hline \multirow[t]{5}{*}{ Malaria intervention } & ITN/LLIN ownership/coverage/use & 19 & 323342435766747599100103112113119122123126129134 \\
\hline & IRS & 8 & 2633747599100112117 \\
\hline & ACTs & 5 & 749899119129 \\
\hline & Treatment seeking rate & 3 & 66119129 \\
\hline & Reporting and testing & 1 & 66 \\
\hline None & None & 19 & $194650-53566268105114120121124125127$ 136-138 \\
\hline
\end{tabular}

ACTs, Artemisinin-based combined therapy; CTI, Compound topographic index; DEM, Digital elevation models; EIR, Entomological inoculation rate; EVI, Enhanced vegetation index; GIS, geographical information system; IRS, Indoor residual spraying; ITN, Insecticidetreated bed nets; LLIN, Long lasting insecticidal nets; LST, Land surface temperature; NDVI, Normalised difference vegetation index; NDWI, Normalised difference water index; SES, Social economic status; TSI, Temperature suitability index; TWI, Topographic wetness index.

local Moran's I statistic and the Global Moran's I statistic. On the other hand, nine $(8 \%)$ studies used temporal models to explore and forecast malaria risk at different temporal resolutions, with the autoregressive integrated moving average (ARIMA) model used in seven (6\%) studies being the most common. Two studies (2\%) used a univariate seasonal ARIMA model to explore malaria risk patterns (table 2).

The Bayesian spatial only and space-time kriging-a statistically unbiased and robust interpolation method appropriate for study settings with limited data; was used in five $(6 \%)$ studies, to predict risk at unsampled locations,${ }^{67} 72$ improve predictions in geographical areas with considerable variation between observed values and model predictions ${ }^{45} 73$ and model the spatial and temporal correlations of monthly malaria morbidity cases. ${ }^{62}$

Using point-referenced data sourced from multiple independent surveys, 27 (25\%) studies applied both the model-based geostatistical (MBG) and Bayesian MBG methods to analyse, predict and map malaria risk. In this framework, the spatio-temporal dependency was modelled as a Gaussian process in fourteen (13\%) studies. $^{8} 343637434648-505359606474$ Spatial only random effects (dependency) were modelled via the Gaussian prior distribution ${ }^{26} 2729-3141636575-78$ in 12 (11\%) studies. Temporal random effects were assigned a first order autoregressive AR (1) prior distribution in three (3\%) studies $^{37} 7579$ and second order autoregressive $\operatorname{AR}(2)$ prior distribution ${ }^{8}$ in one ( $1 \%$ ) study (table 4 ).

Using observations aggregated over distinct geographical region/spatial partitions/adjacent units (eg, census tract, administrative boundaries); 15 (14\%) studies used the Bayesian conditional autoregressive (CAR) models, to explore the spatial and spatio-temporal variation of malaria risk. To account for the temporal dependency between consecutive time points; seven studies $(6 \%)$ used the first order autoregressive AR (1) prior process, whereas one study $(1 \%)$ used the random walk of order one RW (1) prior process (table 4).

\section{Other models}

Generalised linear modelling framework, such as the Poisson, logistic regression, negative binomial and geographically weighted regression, was used in fifteen studies (14\%). These models explored the association of malaria counts or rates and its correlates, using appropriate exponential distribution families. Machine learning techniques such as the artificial neural network and the boosted regression tree were used to analyse incidence patterns and to examine malaria prevalence, respectively (table 4).

\section{Model validation, performance and uncertainty}

A range of different validation techniques were used to assess model fitness and to select the optimal predictive 
Table 4 Structure of the spatio-temporal models

\begin{tabular}{|c|c|c|c|c|c|}
\hline ID & References & Year & Space & Time & Space time \\
\hline 1 & Abellana et $a l^{84}$ & 2008 & CAR & & \\
\hline 2 & Alegana et $a l^{64}$ & 2016 & Markov random field & - & Gaussian \\
\hline 3 & Alegana et $a^{25}$ & 2013 & - & - & CAR \\
\hline 4 & Alemu et $a l^{51}$ & 2013 & - & Temporal trend - ARIMA & - \\
\hline 5 & Amek et $a l^{79}$ & 2012 & Gaussian & $\mathrm{AR}(1)$ & - \\
\hline 6 & Amratia et $a l^{61}$ & 2019 & Gaussian & - & - \\
\hline 7 & Appiah et $a /^{62}$ & 2011 & - & - & STOK \\
\hline 8 & Awine et $a l^{133}$ & 2018 & - & - & SARIMA \\
\hline 9 & Bejon et $a l^{39}$ & 2010 & Cluster analysis & Temporal trends & - \\
\hline 10 & Bejon et $a l^{105}$ & 2014 & Cluster analysis & - & - \\
\hline 11 & Belay et $a l^{106}$ & 2017 & - & Temporal trends & - \\
\hline 12 & Bennett et al ${ }^{60}$ & 2013 & - & - & Gaussian \\
\hline 13 & Bennett et $a l^{75}$ & 2016 & Gaussian & - & - \\
\hline 14 & Bennett et al ${ }^{66}$ & 2014 & CAR & CAR & CAR \\
\hline 15 & Bhatt et $a l^{74}$ & 2015 & Markov random field & $\mathrm{AR}(1)$ & Gaussian \\
\hline 16 & Bisanzio et al ${ }^{113}$ & 2015 & Markov random field & B - splines with RW (2) & - \\
\hline 17 & $\mathrm{BM} \& \mathrm{OE}^{44}$ & 2007 & CAR & - & - \\
\hline 18 & Bousema et $\mathrm{al}^{123}$ & 2010 & Hotspot analysis & - & - \\
\hline 19 & Ceccato et al $^{71}$ & 2007 & Cluster analysis & - & - \\
\hline 20 & Chipeta et $a l^{50}$ & 2019 & - & - & Gaussian \\
\hline 21 & Chirombo et al $^{109}$ & 2020 & Markov random field & Markov random field & Gaussian \\
\hline 22 & Cissoko et $\left.a\right|^{38}$ & 2020 & Cluster analysis & Temporal trend & \\
\hline 23 & Colborn et $a /^{88}$ & 2018 & - & - & Gaussian \\
\hline 24 & Coulibaly et $a l^{54}$ & 2013 & Cluster analysis & - & - \\
\hline 25 & DePina et $a^{118}$ & 2019 & Cluster analysis & Temporal trend & - \\
\hline 26 & Diboulo et al ${ }^{41}$ & 2016 & Gaussian & - & - \\
\hline 27 & Ferrão et $a^{70}$ & $2017 a$ & - & Temporal trend - ARIMA & - \\
\hline 28 & Ferrão et $a l^{69}$ & $2017 b$ & - & Temporal trend - ARIMA & - \\
\hline 29 & Ferrari et $a^{124}$ & 2016 & Cluster analysis & - & - \\
\hline 30 & Gaudart et al ${ }^{125}$ & 2006 & Cluster analysis & Temporal trend - ARIMA & - \\
\hline 31 & Gemperli et al ${ }^{67}$ & 2006 & $\begin{array}{l}\text { Exponential correlation } \\
\text { function }\end{array}$ & - & - \\
\hline 32 & Gething et al ${ }^{98}$ & 2016 & - & P - splines with RW (1) & - \\
\hline 33 & Giardina et $\mathrm{al}^{78}$ & 2015 & Gaussian & - & - \\
\hline 34 & Giardina et $a l^{65}$ & 2012 & Multivariate Normal & - & - \\
\hline 35 & Giardina et $a l^{101}$ & 2014 & Gaussian & - & - \\
\hline 36 & Giorgi et al ${ }^{46}$ & 2018 & - & - & Gaussian \\
\hline 37 & Gómez-Barroso et al ${ }^{20}$ & 2017 & Cluster analysis & - & - \\
\hline 38 & Gosoniu et $a^{27}$ & 2012 & Gaussian & - & - \\
\hline 39 & Gosoniu et $a l^{76}$ & 2010 & Gaussian & - & - \\
\hline 40 & Gosoniu et $a l^{63}$ & 2006 & Gaussian & - & - \\
\hline 41 & Houngbedji et $a l^{77}$ & 2016 & Normal & - & - \\
\hline 42 & Ihantamalala et al ${ }^{114}$ & 2018 & Cluster analysis & - & - \\
\hline 43 & Ikeda et $a l^{116}$ & 2017 & - & - & SOM \\
\hline 44 & Ishengoma et al $\left.\right|^{126}$ & 2018 & - & Temporal trends & - \\
\hline
\end{tabular}


BMJ Global Health

Table 4 Continued

\begin{tabular}{|c|c|c|c|c|c|}
\hline ID & References & Year & Space & Time & Space time \\
\hline 45 & Kabaghe et al ${ }^{43}$ & 2017 & Gaussian & - & - \\
\hline 46 & Kabaria et $a l^{55}$ & 2016 & - & - & BRT \\
\hline 47 & Kamuliwo et al $^{117}$ & 2015 & Cluster analysis & - & - \\
\hline 48 & Kang et $a l^{37}$ & 2018 & Gaussian & $\mathrm{AR}(1)$ & - \\
\hline 49 & Kangoye et $a^{127}$ & 2016 & Cluster analysis & - & - \\
\hline 50 & Kanyangarara et $a l^{28}$ & 2016 & - & - & - \\
\hline 51 & Kazembe et $\left.a\right|^{31}$ & 2006 & Gaussian & - & - \\
\hline 52 & Kifle et $a l^{107}$ & 2019 & Cluster analysis & Temporal trends - SARIMA & \\
\hline 53 & Kigozi et al ${ }^{128}$ & 2016 & - & Temporal trend- ARIMA & - \\
\hline 54 & Kleinschmidt et al ${ }^{45}$ & 2000 & Kriging & - & - \\
\hline 55 & Kleinschmidt et $\mathrm{al}^{73}$ & $2001 a$ & Kriging & - & - \\
\hline 56 & Kleinschmidt et al $/^{97}$ & $2001 b$ & Kriging & - & - \\
\hline 57 & Kleinschmidt et al $^{136}$ & 2002 & Normal & Normal & - \\
\hline 58 & Mabaso et al ${ }^{131}$ & 2005 & CAR & - & $\mathrm{AR}(1)$ \\
\hline 59 & Mabaso et $\left.a\right|^{24}$ & 2006 & CAR & $\mathrm{AR}(1)$ & - \\
\hline 60 & Macharia et $a l^{53}$ & 2018 & - & - & Gaussian \\
\hline 61 & Mfueni et $a l^{52}$ & 2018 & - & - & - \\
\hline 62 & Midekisa et $a l^{130}$ & 2012 & - & Temporal trend - SARIMA & - \\
\hline 63 & Millar et $a l^{100}$ & 2018 & - & - & - \\
\hline 64 & Mirghani et al ${ }^{120}$ & 2010 & Cluster analysis & - & - \\
\hline 65 & Mlacha et $a l^{56}$ & 2017 & Cluster analysis & - & - \\
\hline 66 & Mukonka et al ${ }^{138}$ & 2014 & - & Temporal trends & - \\
\hline 67 & Mukonka et al ${ }^{115}$ & 2015 & Cluster analysis & - & - \\
\hline 68 & Mwakalinga et al ${ }^{121}$ & 2016 & Cluster analysis & - & - \\
\hline 69 & Ndiath et $a l^{57}$ & 2015 & Cluster analysis & - & - \\
\hline 70 & Ndiath et $a l^{42}$ & 2014 & Cluster analysis & - & - \\
\hline 71 & Nguyen et al ${ }^{110}$ & 2020 & Gaussian & - & Gaussian \\
\hline 72 & Noor et $a l^{48}$ & $2013 a$ & Gaussian & - & GRF \\
\hline 73 & Noor et $a l^{34}$ & 2008 & Gaussian & - & - \\
\hline 74 & Noor et $a l^{58}$ & $2012 b$ & - & - & GRF \\
\hline 75 & Noor et $a l^{36}$ & 2009 & - & - & GRF \\
\hline 76 & Noor et $a l^{8}$ & 2014 & Gaussian & $\mathrm{AR}(2)$ & - \\
\hline 77 & Noor et $a l^{49}$ & $2013 b$ & - & - & GRF \\
\hline 78 & Noor et $a l^{59}$ & $2012 a$ & Gaussian & - & $\begin{array}{l}\text { Stationary } \\
\text { Gaussian }\end{array}$ \\
\hline 79 & Nyadanu et $a l^{108}$ & 2019 & Cluster analysis & - & - \\
\hline 80 & Okunola et $\left.a\right|^{23}$ & 2019 & Cluster analysis & - & - \\
\hline 81 & Onyiri ${ }^{29}$ & 2015 & Gamma & - & - \\
\hline 82 & Ouedraogo et al ${ }^{40}$ & 2018 & - & Temporal trend- ARIMA & - \\
\hline 83 & Ouédraogo et al ${ }^{111}$ & 2020 & CAR & AR (1) / Temporal trends & \\
\hline 84 & Peterson et $a l^{134}$ & 2009 & Cluster analysis & - & - \\
\hline 85 & Pinchoff et $\left.a\right|^{35}$ & 2015 & - & - & - \\
\hline 86 & Raso et $a /^{30}$ & 2012 & Multivariate Normal & - & - \\
\hline 87 & Rouamba et $a l^{102}$ & 2020 & CAR & CAR & Gaussian \\
\hline 88 & Rumisha et al ${ }^{80}$ & 2014 & Gaussian & $\mathrm{AR}(1)$ & - \\
\hline
\end{tabular}

Continued 


\begin{tabular}{|c|c|c|c|c|c|}
\hline ID & References & Year & Space & Time & Space time \\
\hline 89 & Selemani et $a l^{32}$ & 2015 & Cluster analysis & - & - \\
\hline 90 & Selemani et $a l^{103}$ & 2016 & CAR & $\operatorname{AR}(1)$ & - \\
\hline 91 & Sewe et $a l^{104}$ & 2016 & - & Natural cubic spline & - \\
\hline 92 & Seyoum et $a^{137}$ & 2017 & Cluster analysis & - & - \\
\hline 93 & Shaffer et $a^{122}$ & 2020 & Cluster analysis & Temporal trends & - \\
\hline 94 & Simon et al ${ }^{112}$ & 2013 & Cluster analysis & - & - \\
\hline 95 & Siraj et $a l^{135}$ & 2015 & CAR & - & - \\
\hline 96 & Snow et $a l^{19}$ & 2017 & CAR & CAR & - \\
\hline 97 & Snow et $a l^{132}$ & 1998 & - & - & - \\
\hline 98 & Solomon et $a l^{33}$ & 2019 & Cluster analysis & - & - \\
\hline 99 & Ssempiira et al $^{119}$ & $2018 a$ & CAR & AR (1) / temporal trend & - \\
\hline 100 & Ssempiira et $a l^{129}$ & $2018 b$ & CAR & AR (1) / temporal trend & - \\
\hline 101 & Ssempiira et $a^{99}$ & $2017 b$ & CAR & - & - \\
\hline 102 & Ssempiira et $a l^{72}$ & $2017 a$ & - & - & - \\
\hline 103 & Sturrock et $a l^{47}$ & 2014 & CAR & Temporal trend & - \\
\hline 104 & Yankson et $\mathrm{al}^{26}$ & 2019 & Gaussian & - & - \\
\hline 105 & Yeshiwondim et $a l^{68}$ & 2009 & - & - & - \\
\hline 106 & Zacarias and Andersson ${ }^{22}$ & 2011 & CAR & $\mathrm{AR}(1)$ & - \\
\hline 107 & Zacarias and Majlender ${ }^{21}$ & 2011 & CAR & $\mathrm{RW}(1)$ & - \\
\hline
\end{tabular}

AR, autoregressive; ARIMA, autoregressive integrated moving average; BRT, boosted regression tree; CAR, conditional autoregressive; GRF, Gaussian random field; RW, random walk; SARIMA, seasonal autoregressive integrated moving average; SOM, self-organising maps; STOK, space-time ordinary kriging.

models. The most commonly used approach entailed partitioning the data for model training and validation and was employed in $24(22 \%)$ studies. The training set was then used to validate the predictive model fit, whereas the validation set was used for assessing the model predictive ability. The representative holdout datasets were selected using a spatially and temporal declustered algorithm, 8484959 stratified sampling approach $^{75}$ and randomly. ${ }^{3546505380}$ Information criteria, that is, the deviance information criterion, Akaike information criterion and the BIC were used in seventeen $(16 \%)$ studies. Seven (7\%) studies used variogram-based algorithms to identify estimates falling within the $95 \%$ credible interval. Model precision and accuracy metrics included the mean prediction error, root mean squared error, mean absolute prediction error, mean error and the SD (table 2).

\section{Summarised modelling framework}

The rapid expansion of methods and data informs the need to guide future spatial and spatio-temporal modelling of infectious diseases in SSA (online supplemtal table 4). We illustrate a framework composed of four fundamental modelling entities, namely: inputs, process, stochastic components and output. Malaria data and covariates sourced from different spatial and temporal resolutions are considered to be the model inputs. A series of progressive and interdependent steps/processes on the model inputs are then used to generate the outputs (posterior marginals). Posterior marginals can then be approximated using iterative computational techniques such as the Markov Chain Monte Carlo methods or by using numerical integrations via the Integrated Nested Laplace Approximations method. ${ }^{81}{ }^{82}$ (figure 4$)$

\section{DISCUSSION}

Scalable guidelines for rigorous and transparent statistical methodology are necessary for reproducible malaria risk estimation. This review offers a comprehensive appraisal and synthesis of methods and covariates used in malaria risk mapping in SSA in the last five decades.

\section{Sources of malaria data}

High-resolution maps revealing the spatio-temporal variation of malaria endemicity are useful for estimating malaria burden, quantifying the effectiveness of control initiatives and assessing the progress towards its elimination nationally and subnationally. However, malaria risk mapping efforts in SSA are rarely based on routinely collected data. Instead, periodic and costly household survey's data have traditionally been used in modelling malaria risk. To address this challenge and obtain robust estimates reflective of the subnational burden, WHO initiated the high burden to high impact approach in 2018, which underscored the need for reliable national data systems. This is considered central to the understanding 


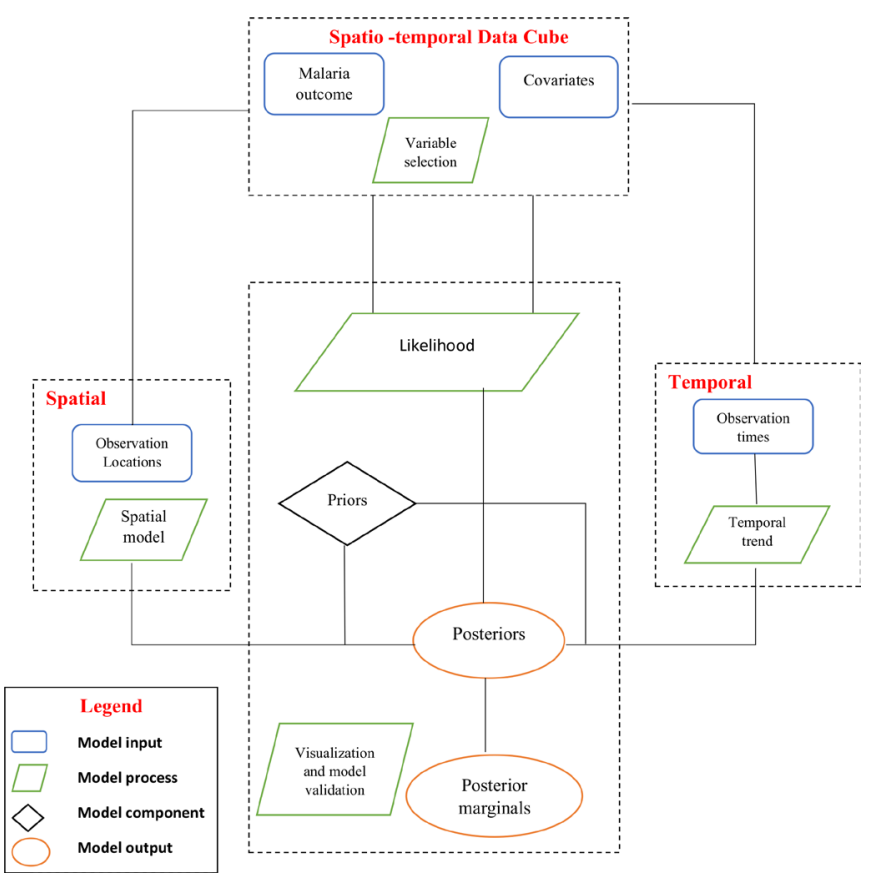

Figure 4 Schematic illustration of the spatio-temporal modelling framework for malaria risk in sub-Saharan Africa.

of malaria burden in low transmission settings and in the most vulnerable populations. ${ }^{3}$ Additionally, the approach has availed more malaria data in malaria-endemic settings, and caution is needed when gathering and interpreting findings generated from data at fine spatial and/ or temporal scales with varying degree of completeness and representativeness. ${ }^{83}$

The steady growth of satellite, remote sensing platforms and curated databases has made available a rich suite of both environmental and socioeconomic covariates at a finer level of detail useful for mapping malaria risk at high spatial and temporal resolution. Validating the quality of available satellite data prior to their inclusion in malaria studies remains central to achieving robust estimates.

While malaria incidence and prevalence metrics can be modelled from routine health information systems and sample surveys respectively, caution should be taken when interpreting estimates as both metrics are products of interacting factors such as interventions, sociodemographic and environmental factors that may contribute to the overall risk. A concise picture may be achieved by measuring malaria indicators at a finer spatial scale and exploring the nature and scope of the interaction. Data on malaria mortality as an outcome were sparse, and efforts must be made to increase data collection and improve the sensitivity and specificity of malaria mortality burden attribution in SSA. ${ }^{74}$ As many countries in SSA transition epidemiologically from high to low malaria transmission zones, obtaining useful metrics for mapping risk from sparse national surveys at low, moderate and heterogeneous transmission settings possess unique challenges for measuring progress and impact. ${ }^{84}$
The paucity of continuous, reliable data necessary to yield estimates with greater geographical and temporal richness is a growing concern in the era of evidencebased public health. A high-quality, routinely collected data avail an alternative source of malaria metrics for continuous analysis over time. ${ }^{83}$ Investments should be channelled towards establishing and addressing inadequacies in the health information systems to enable subnational mapping of malaria risk. However, the urge for quality data and the increasing need of accurate estimates can significantly be improved by adoption of data-driven modelling approaches that leverage both routine and household survey data in their model framework. ${ }^{85}$ Additionally, detailed information on critical data sources, preliminary data adjustments undertaken before modelling should be availed as an important step towards enhancing reproducibility of methods and estimates. ${ }^{86}$

\section{Understanding covariates used in mapping malaria risk}

Improving the precision of malaria risk estimates largely depends on limiting subjective decisions. These decisions may impact on the modelling process, even as more covariates becomes accessible at finer geographical and temporal resolutions. Studies have shown large variables to be desirable for prediction, whereas small sets of variables to be meaningful for inference. ${ }^{87}$ It is important to understand the complex relationship between covariates at the same spatial or temporal resolution, to avoid overfitting. The trade-off between model interpretability, predictive ability, spatial and temporal scope, data accessibility and computational limitations are critical factors worth considering when selecting candidate covariates. Evidentially variable selection is an important preliminary step for a robust malaria risk mapping exercise. However, this approach continues to receive little attention amidst the growing diversity of covariate layers that need to be identified and included in the models.

Environmental and climatic factors influence mosquito vector abundance, distribution and longevity; at different time scales ${ }^{88}$ and are important for mapping malaria risk. ${ }^{59} \mathrm{~A}$ scoping review by Zinszer $e t a l^{89}$ highlighted the importance of climatic-related predictors in malaria risk prediction. Understanding the different facets and extent of how climatic influences on malaria risk variation; has been enhanced by advances in remote sensing and satellite imagery technology, increasing the availability of remotely sensed climatic data at high spatial resolution. ${ }^{35}$ In this review proxies of; temperature (land surface temperature, temperature suitability index, mean $/ \mathrm{min} / \mathrm{max} /$ weekly) and rainfall/precipitation (weekly/monthly/annual) were the most widely used environmental factors related to malaria transmission in SSA. Vegetative indices such as elevation, surface moisture, land use and land cover were included in malaria risk maps, primarily due to their association with temperature and precipitation which indirectly influences $^{90}$ the distribution of malaria. Remote sensing will continue to feature prominently as a cost-effective tool 
for mapping malaria risk in SSA and an important source of environmental and climatic covariates. ${ }^{91}$ The review further demonstrates the significance of non-climatic determinants such as malaria interventions and demographic factors in malaria risk mapping.

\section{Modelling frameworks in malaria risk mapping}

Complex decisions involving key modelling components such as covariates to include, preliminary data preprocessing and diagnostics checks demands advanced statistical knowledge. Extensive computational algorithms and complex spatio-temporal data structures may limit the applicability of these modelling approaches to experts. Furthermore, complex models used to represent malaria heterogeneity may not necessarily represent the truth on the ground. Thus, the statistical uncertainties around model estimates should be carefully examined, and the varying quantities and quality malaria data, that informs modelling approaches accounted for.

The review highlights the prominence and flexibility of geostatistical methods in modelling spatial and spatiotemporal malaria patterns, at policy-relevant units and thresholds. ${ }^{46}$ Geostatistical methods provide a useful framework for interpolating imperfect data from multiple independent surveys by estimating spatial dependence from the data. At low spatial resolution, the Bayesian geostatistical framework accounts for uncertainty resulting from sparsely sampled point-referenced data by assigning priors that allows 'borrowing of strength' from adjacent regions leading to robust estimates and predictions. ${ }^{92}$ Amidst the current scarcity and imperfections of routine, high resolution and spatially expansive malaria data in many SSA countries, using geostatistical methods with data from multiple independent georeferenced surveys, continues to be important for generating reliable estimates.

Bayesian hierarchical CAR models are useful for modelling spatially correlated areal data by smoothing noisy estimates and leveraging information from adjacent regions. However, choosing an appropriate prior specification for the parameters defining the spatial interaction is inevitable and sometimes challenging. Notably, the spatial dependence among neighbouring regions is accounted for by assuming a CAR process in the random effects. For example, in the Besag York and Mollie/ convolution model, location-specific spatial effects are assumed to follow a normal distribution with the mean equal to the average of its neighbours and the variance considered to be inversely proportional to the number of neighbours. In the Leroux et al model, the spatial dependence is based on the weighted average of both the independent random effects and spatially structured random effects. ${ }^{93}{ }^{94}$ The intrinsic CAR and Besag, York and Mollie (BYM) were the most frequent global spatial smoothing specifications used in the review; given their easy implementation in a range of softwares. However, caution should be taken to minimise over smoothing-obscuring the underlying geographical patterns. Future modelling studies should compare the impact of using other spatial smoothing priors.

Overall, malaria risk mapping has increased dramatically over the last decades, with novel methods advanced to meet the quest for accurate estimates of malaria burden. Whereas most approaches are built on classical statistical methods, recent advances in computing, availability of geographically referenced data have ushered/propagated new techniques designed to address existing challenges. These approaches include ensemble modelling, neural networks, simulation-based methods and bootstrap models to better capture space-time interactions.

\section{Recommendations for best practices}

As malaria landscape diversifies in the next decade, investments in primary data collection at subnational scales, development and continuous application of robust modelling tools will continue to be important priorities in malaria control and elimination efforts. In the era of open data policy and reproducible research, our review reiterates the importance of periodically reviewing, validating and updating malaria maps to accommodate new data sources, improved data quality, enhanced computing power and novel methodological approaches. Variable selection procedures should be data driven and objectively developed to the maximise the predictive accuracy of malaria risk mapping. The spatio-temporal modelling framework should incorporate practical challenges facing control and elimination of malaria in SSA. These challenges are: human migration within and among endemic zones, mapping asymptotic infection reservoirs and accounting for differential immunity within a population. ${ }^{95} 96$

\section{Strengths and limitations}

The review search strategy was exhaustive and transparent, in accordance with the current methodological guidelines and included studies have provided a fair depiction of malaria risk mapping efforts in SSA. The methodological approach of the included studies was diverse, making meta-analysis inappropriate. The review considered only studies published in English and relevant papers published in other languages might have been excluded.

\section{CONCLUSIONS}

Malaria risk mapping remains an important component for understanding the burden of malaria in SSA. The review has described modelling approaches and examined covariates used in mapping malaria risk in different epidemiological contexts. As malaria transmission continues to decline in SSA, the use of metrics that accurately describes changes in its transmission intensity across space and time will be important for the design and implementation of evidence-based control and elimination measures. 


\section{Author affiliations}

${ }^{1}$ Discipline of Public Health Medicine, University of KwaZulu-Natal, Durban, South Africa

${ }^{2}$ Faculty of Agriculture and Natural Resources, University of Namibia, Windhoek, Namibia

${ }^{3}$ Population Health Unit, Kenya Medical Research Institute-Wellcome Trust Research Programme, Nairobi, Kenya

${ }^{4}$ Centre for Tropical Medicine and Global Health, Nuffield Department of Clinical Medicine, University of Oxford, Oxford, UK

${ }^{5}$ Department of Disease Control, Faculty of Infectious and Tropical Diseases, London School of Hygiene and Tropical Medicine, London, UK

\section{Twitter Peter M Macharia @Pete_M_M}

Acknowledgements The authors thank the College of Health Sciences, systematic review services and library services at the University of KwaZulu-Natal for providing training and resources at the initial phases of the review.

Contributors JNO, BS and RWS conceived and designed the systematic review. JNO, BS and CK conducted the literature search, study selection and data extraction. JNO wrote the first draft of the manuscript with assistance from CK and BS. PMM and RWS revised the draft critically for important intellectual content. All authors read and approved the final version of the manuscript.

Funding JNO acknowledges support from the University of KwaZulu Natal, College of Health Sciences postgraduate scholarship scheme. RWS is supported as a Wellcome Trust Principal Fellow (\#103602 and 212176) that also supported PMM. PMM acknowledges support for his PhD under the IDeALs Project part of the DELTAS Africa Initiative (DEL-15-003). The DELTAS Africa Initiative is an independent funding scheme of the African Academy of Sciences (AAS)'s Alliance for Accelerating Excellence in Science in Africa and supported by the New Partnership for Africa's Development Planning and Coordinating Agency with funding from the Wellcome Trust (107769) and the UK government. RWS and PMM are grateful to the support of the Wellcome Trust to the Kenya Major Overseas Programme (203077).

Competing interests None declared.

Patient and public involvement Patients and/or the public were not involved in the design, or conduct, or reporting, or dissemination plans of this research.

Patient consent for publication Not required.

Provenance and peer review Not commissioned; externally peer reviewed.

Data availability statement All data relevant to the study are included in the article or uploaded as online supplemental information. The data supporting conclusions made in this review are available in the detailed reference list.

Open access This is an open access article distributed in accordance with the Creative Commons Attribution Non Commercial (CC BY-NC 4.0) license, which permits others to distribute, remix, adapt, build upon this work non-commercially, and license their derivative works on different terms, provided the original work is properly cited, appropriate credit is given, any changes made indicated, and the use is non-commercial. See: http://creativecommons.org/licenses/by-nc/4.0/.

\section{ORCID iDs}

Julius Nyerere Odhiambo http://orcid.org/0000-0002-9817-1849

Peter M Macharia http://orcid.org/0000-0003-3410-188

\section{REFERENCES}

1 World Health Organization. Health in the 2030 agenda for sustainable development. World health assembly resolution 6911 Geneva; 2016.

2 World Health Organization. Global technical strategy for malaria 2016-2030; 2015.

3 World Health Organization. World malaria report 2019. Geneva; 2019.

4 Snow RW, Noor AM. Malaria risk mapping in Africa: The historica context to the Information for Malaria (INFORM) project. Nairobi, Kenya: Working paper in support of the INFORM Project funded by the Department for International Development and The Wellcome Trust, UK; 2015.

5 Snow RW, Marsh K, le Sueur D. The need for maps of transmission intensity to guide malaria control in Africa. Parasitology Today 1996;12:455-7.
6 Le Sueur D, Binka F, Lengeler C, et al. An atlas of malaria in Africa. Africa health 1997;19:23-4.

7 Omumbo JA, Noor AM, Fall IS, et al. How well are malaria maps used to design and finance malaria control in Africa? PLoS One 2013;8:e53198.

8 Noor AM, Kinyoki DK, Mundia CW, et al. The changing risk of Plasmodium falciparum malaria infection in Africa: 2000-10: a spatial and temporal analysis of transmission intensity. The Lancet 2014;383:1739-47.

9 Kraemer MUG, Hay SI, Pigott DM, et al. Progress and challenges in infectious disease cartography. Trends Parasitol 2016;32:19-29.

$10 \mathrm{Ye} \mathrm{Y,} \mathrm{Andrada} \mathrm{A.} \mathrm{Estimating} \mathrm{malaria} \mathrm{incidence} \mathrm{through} \mathrm{modeling} \mathrm{is}$ a good academic exercise, but how practical is it in high-burden settings? Am J Trop Med Hyg 2020;102:701-2.

11 Weiss DJ, Mappin B, Dalrymple U, et al. Re-Examining environmental correlates of Plasmodium falciparum malaria endemicity: a data-intensive variable selection approach. Malar J 2015;14:68

12 Dalrymple U, Mappin B, Gething PW. Malaria mapping: understanding the global endemicity of falciparum and vivax malaria. BMC Med 2015;13:140.

13 Canelas T, Castillo-Salgado C, Ribeiro H. Systematized literature review on spatial analysis of environmental risk factors of malaria transmission. Adv Infect Dis 2016;06:52-62.

14 Odhiambo JN, Sartorius B. Spatio - temporal modelling assessing the burden of malaria in affected low and middle-income countries: a scoping review. BMJ Open 2018;8:e023071.

15 Liberati Aet al. The PRISMA statement for reporting systematic reviews and meta-analyses of studies that evaluate health care interventions: explanation and elaboration. Ann Intern Med 2009:151:W-W-94.

16 Moher Det al. Preferred reporting items for systematic reviews and meta-analyses: the PRISMA statement. Ann Intern Med 2009;151:264-9.

17 World Health Organization. World malaria report 2015. Geneva; 2016.

18 Aswi A, Cramb SM, Moraga P, et al. Bayesian spatial and spatiotemporal approaches to modelling dengue fever: a systematic review. Epidemiol Infect 2019;147.

19 Snow RW, Sartorius B, Kyalo D, et al. The prevalence of Plasmodium falciparum in sub-Saharan Africa since 1900. Nature 2017;550:515-8.

20 Gómez-Barroso D, García-Carrasco E, Herrador Z, et al. Spatial clustering and risk factors of malaria infections in Bata district, equatorial guinea. Malar J 2017;16:146.

21 Zacarias OP, Majlender P. Comparison of infant malaria incidence in districts of Maputo Province, Mozambique. Malar J 2011:10:93.

22 Zacarias OP, Andersson M. Spatial and temporal patterns of malaria incidence in Mozambique. Malar J 2011;10:189.

23 Okunlola OA, Oyeyemi OT. Spatio-Temporal analysis of association between incidence of malaria and environmental predictors of malaria transmission in Nigeria. Sci Rep 2019;9:1-11.

24 Mabaso MLH, Vounatsou P, Midzi S, et al. Spatio-Temporal analysis of the role of climate in inter-annual variation of malaria incidence in Zimbabwe. Int J Health Geogr 2006;5:20.

25 Alegana VA, Atkinson PM, Wright JA, et al. Estimation of malaria incidence in northern Namibia in 2009 using Bayesian conditionalautoregressive spatial-temporal models. Spat Spatiotemporal Epidemiol 2013;7:25-36.

26 Yankson R, Anto EA, Chipeta MG. Geostatistical analysis and mapping of malaria risk in children under 5 using point-referenced prevalence data in Ghana. Malar J 2019;18:67.

27 Gosoniu L, Msengwa A, Lengeler C, et al. Spatially explicit burden estimates of malaria in Tanzania: Bayesian geostatistical modeling of the malaria indicator survey data. PLoS One 2012;7:e23966.

28 Kanyangarara M, Mutambu S, Kobayashi T, et al. High-Resolution Plasmodium falciparum malaria risk mapping in Mutasa district, Zimbabwe: implications for regaining control. Am J Trop Med Hyg 2016;95:141-7.

29 Onyiri N. Estimating malaria burden in Nigeria: a geostatistical modelling approach. Geospat Health 2015;10.

30 Raso G, Schur N, Utzinger J, et al. Mapping malaria risk among children in Côte d'Ivoire using Bayesian geo-statistical models. Malar J 2012;11:160.

31 Kazembe LN, Kleinschmidt I, Holtz TH, et al. Spatial analysis and mapping of malaria risk in Malawi using point-referenced prevalence of infection data. Int J Health Geogr 2006;5:41.

32 Selemani M, Mrema S, Shamte A, et al. Spatial and space-time clustering of mortality due to malaria in rural Tanzania: evidence from Ifakara and Rufiji health and demographic surveillance system sites. Malar J 2015;14:369. 
33 Solomon T, Loha E, Deressa W, et al. Spatiotemporal clustering of malaria in southern-central Ethiopia: a community-based cohort study. PLoS One 2019;14:e0222986.

34 Noor AM, Clements ACA, Gething PW, et al. Spatial prediction of Plasmodium falciparum prevalence in Somalia. Malar J 2008;7:159.

35 Pinchoff J, Chaponda M, Shields T, et al. Predictive malaria risk and uncertainty mapping in Nchelenge district, Zambia: evidence of widespread, persistent risk and implications for targeted interventions. Am J Trop Med Hyg 2015;93:1260-7.

36 Noor AM, Gething PW, Alegana VA, et al. The risks of malaria infection in Kenya in 2009. BMC Infect Dis 2009;9:180

37 Kang SY, Battle KE, Gibson HS, et al. Spatio-temporal mapping of Madagascar's Malaria Indicator Survey results to assess Plasmodium falciparum endemicity trends between 2011 and 2016 . BMC Med 2018;16:71.

38 Cissoko M, Sagara I, Sankaré MH, et al. Geo-epidemiology of malaria at the health area level, dire health district, Mali, 20132017. Int J Environ Res Public Health 2020;17:3982-16.

39 Bejon P, Williams TN, Liljander A, et al. Stable and unstable malaria hotspots in longitudinal cohort studies in Kenya. PLoS Med 2010;7:e1000304.

40 Ouedraogo B, Inoue Y, Kambiré A, et al. Spatio-Temporal dynamic of malaria in Ouagadougou, Burkina Faso, 2011-2015. Malar J 2018;17:138.

41 Diboulo E, Sié A, Vounatsou P. Assessing the effects of malaria interventions on the geographical distribution of parasitaemia risk in Burkina Faso. Malar J 2016;15:228.

42 Ndiath M, Faye B, Cisse B, et al. Identifying malaria hotspots in Keur SOCE health and demographic surveillance site in context of low transmission. Malar J 2014;13:453.

43 Kabaghe AN, Chipeta MG, McCann RS, et al. Adaptive geostatistical sampling enables efficient identification of malaria hotspots in repeated cross-sectional surveys in rural Malawi. PLoS One 2017;12:e0172266

44 BM D, OE A. Spatial association between malaria pandemic and mortality. Data Science Journal 2007;6:145-53.

45 Kleinschmidt I, Bagayoko M, Clarke GPY, et al. A spatial statistical approach to malaria mapping. Int J Epidemiol 2000;29:355-61.

46 Giorgi E, Osman AA, Hassan AH, et al. Using non-exceedance probabilities of policy-relevant malaria prevalence thresholds to identify areas of low transmission in Somalia. Malar J 2018;17:88.

47 Sturrock HJW, Cohen JM, Keil P, et al. Fine-Scale malaria risk mapping from routine aggregated case data. Malar $J$ 2014;13:421.

48 Noor AM, Alegana VA, Kamwi RN, et al. Malaria control and the intensity of Plasmodium falciparum transmission in Namibia 1969-1992. PLoS One 2013;8:e63350.

49 Noor AM, Uusiku P, Kamwi RN, et al. The receptive versus current risks of Plasmodium falciparumtransmission in northern Namibia: implications for elimination. BMC Infect Dis 2013;13:184.

50 Chipeta MG, Giorgi E, Mategula D, et al. Geostatistical analysis of Malawi's changing malaria transmission from 2010 to 2017. Wellcome Open Res 2019;4:57.

51 Alemu K, Worku A, Berhane Y. Malaria infection has spatial, temporal, and spatiotemporal heterogeneity in unstable malaria transmission areas in Northwest Ethiopia. PLoS One 2013;8:e79966.

52 Mfueni E, Devleesschauwer B, Rosas-Aguirre A, et al. True malaria prevalence in children under five: Bayesian estimation using data of malaria household surveys from three sub-Saharan countries. Malar J 2018;17:65

53 Macharia PM, Giorgi E, Noor AM, et al. Spatio-Temporal analysis of Plasmodium falciparum prevalence to understand the past and chart the future of malaria control in Kenya. Malar J 2018;17:340.

54 Coulibaly D, Rebaudet S, Travassos M, et al. Spatio-Temporal analysis of malaria within a transmission season in Bandiagara, Mali. Malar J 2013;12:82.

55 Kabaria CW, Molteni F, Mandike R, et al. Mapping intra-urban malaria risk using high resolution satellite imagery: a case study of Dar es Salaam. Int J Health Geogr 2016;15:26.

56 Mlacha YP, Chaki PP, Malishee AD, et al. Fine scale mapping of malaria infection clusters by using routinely collected health facility data in urban Dar ES Salaam, Tanzania. Geospat Health 2017;12:294

57 Ndiath MM, Cisse B, Ndiaye JL, et al. Application of geographically-weighted regression analysis to assess risk factors for malaria hotspots in Keur SOCE health and demographic surveillance site. Malar J 2015;14:463.

58 Noor AM, EIMardi KA, Abdelgader TM, et al. Malaria risk mapping for control in the Republic of Sudan. Am J Trop Med Hyg 2012;87:1012-21.
59 Noor AM, Alegana VA, Patil AP, et al. Mapping the receptivity of malaria risk to plan the future of control in Somalia. BMJ Open 2012;2:e001160.

60 Bennett A, Kazembe L, Mathanga DP, et al. Mapping malaria transmission intensity in Malawi, 2000-2010. Am J Trop Med Hyg 2013;89:840-9.

61 Amratia P, Psychas P, Abuaku B, et al. Characterizing local-scale heterogeneity of malaria risk: a case study in Bunkpurugu-Yunyoo district in northern Ghana. Malar J 2019;18:81.

62 Appiah SK, Mueller U, Cross J. Spatio-Temporal modelling of malaria incidence for evaluation of public health policy interventions in Ghana. West Africa 2011.

63 Gosoniu L, Vounatsou P, Sogoba N, et al. Bayesian modelling of geostatistical malaria risk data. Geospat Health 2006;1:127-39.

64 Alegana VA, Atkinson PM, Lourenço C, et al. Advances in mapping malaria for elimination: fine resolution modelling of Plasmodium falciparum incidence. Sci Rep 2016;6:29628.

65 Giardina F, Gosoniu L, Konate L, et al. Estimating the burden of malaria in Senegal: Bayesian zero-inflated binomial geostatistical modeling of the MIS 2008 data. PLoS One 2012;7:e32625.

66 Bennett A, Yukich J, Miller JM, et al. A methodological framework for the improved use of routine health system data to evaluate national malaria control programs: evidence from Zambia. Popul Health Metr 2014;12:30.

67 Gemperli A, Sogoba N, Fondjo E, et al. Mapping malaria transmission in West and central Africa. Trop Med Int Health 2006;11:1032-46.

68 Yeshiwondim AK, Gopal S, Hailemariam AT, et al. Spatial analysis of malaria incidence at the village level in areas with unstable transmission in Ethiopia. Int J Health Geogr 2009;8:5

69 Ferrão JL, Mendes JM, Painho M, et al. Malaria mortality characterization and the relationship between malaria mortality and climate in Chimoio, Mozambique. Malar J 2017;16:212.

70 Ferrão JL, Mendes JM, Painho M. Modelling the influence of climate on malaria occurrence in Chimoio Municipality, Mozambique. Parasit Vectors 2017;10:260.

71 Ceccato P, Ghebremeskel T, Jaiteh M, et al. Malaria stratification, climate, and epidemic early warning in Eritrea. Am J Trop Med Hyg 2007;77:61-8.

72 Ssempiira J, Nambuusi B, Kissa J, et al. The contribution of malaria control interventions on spatio-temporal changes of parasitaemia risk in Uganda during 2009-2014. Parasit Vectors 2017;10:450.

73 Kleinschmidt I, Omumbo J, Briet O, et al. An empirical malaria distribution map for West Africa. Trop Med Int Health 2001;6:779-86.

74 Bhatt S, Weiss DJ, Cameron E, et al. The effect of malaria control on Plasmodium falciparum in Africa between 2000 and 2015. Nature 2015;526:207-11.

75 Bennett A, Yukich J, Miller JM, et al. The relative contribution of climate variability and vector control coverage to changes in malaria parasite prevalence in Zambia 2006-2012. Parasit Vectors 2016:9:431

76 Gosoniu L, Veta AM, Vounatsou P. Bayesian geostatistical modeling of malaria indicator survey data in Angola. PLoS One 2010;5:e9322.

77 Houngbedji CA, Chammartin F, Yapi RB, et al. Spatial mapping and prediction of Plasmodium falciparum infection risk among schoolaged children in Côte d'Ivoire. Parasit Vectors 2016;9:494.

78 Giardina F, Franke J, Vounatsou P. Geostatistical modelling of the malaria risk in Mozambique: effect of the spatial resolution when using remotely-sensed imagery. Geospat Health 2015;10.

79 Amek N, Bayoh N, Hamel M, et al. Spatial and temporal dynamics of malaria transmission in rural Western Kenya. Parasit Vectors 2012;5:86.

80 Rumisha SF, Smith T, Abdulla S, et al. Modelling heterogeneity in malaria transmission using large sparse spatio-tempora entomological data. Glob Health Action 2014;7:22682.

81 Spatial data analysis with R-INLA with some extensions; 2015. American statistical association

82 Rue H, Martino S, Chopin N. Approximate Bayesian inference for latent Gaussian models by using integrated nested Laplace approximations. Journal of the Royal Statistical Society: Series B 2009;71:319-92.

83 Alegana VA, Okiro EA, Snow RW. Routine data for malaria morbidity estimation in Africa: challenges and prospects. BMC Med 2020:18:121.

84 Abellana R, Ascaso C, Aponte J, et al. Spatio-seasonal modeling of the incidence rate of malaria in Mozambique. Malar J 2008;7:228.

85 Thawer SG, Chacky F, Runge M, et al. Sub-national stratification of malaria risk in mainland Tanzania: a simplified assembly of survey and routine data. Malar J 2020;19:1-12. 
86 Atun R. Time for a revolution in reporting of global health data. Lancet 2014;384:937-8.

87 Heinze G, Wallisch C, Dunkler D. Variable selection - A review and recommendations for the practicing statistician. Biom $J$ 2018;60:431-49.

88 Colborn KL, Giorgi E, Monaghan AJ, et al. Spatio-Temporal modelling of weekly malaria incidence in children under 5 for early epidemic detection in Mozambique. Sci Rep 2018;8:9238.

89 Zinszer K, Verma AD, Charland K, et al. A scoping review of malaria forecasting: past work and future directions. BMJ Open 2012;2:e001992.

90 Ferrao JL, Niquisse S, Mendes JM, et al. Mapping and modelling malaria risk areas using climate, socio-demographic and clinical variables in Chimoio, Mozambique. Int J Environ Res Public Health 2018;15:795.

91 Kabaria CW, Molteni F, Mandike R, et al. Mapping intra-urban malaria risk using high resolution satellite imagery: a case study of Dar ES Salaam. Int J Health Geogr 2016;15:26.

92 Diggle PJ, Tawn JA. Moyeed RJJotRSSSC. Model-based geostatistics 1998;47:299-350.

93 Lee D. A comparison of conditional autoregressive models used in Bayesian disease mapping. Spat Spatiotemporal Epidemiol 2011;2:79-89.

94 Leroux BG, Lei X, Breslow N. Estimation of disease rates in small areas: a new mixed model for spatial dependence. statistical models in epidemiology, the environment, and clinical trials. Springer 2000:179-91.

95 Aguilar JB, Gutierrez JB. An epidemiological model of malaria accounting for asymptomatic carriers. Bull Math Biol 2020;82:1-55.

96 Kimenyi KM, Wamae K, Ochola-Oyier LI. Understanding P. falciparum Asymptomatic Infections: A Proposition for a Transcriptomic Approach. Front Immunol 2019;10:2398.

97 Kleinschmidt let al. Use of generalized linear mixed models in the spatial analysis of small-area malaria incidence rates in KwaZulu Natal, South Africa. Am J Epidemiol 2001;153:1213-21.

98 Gething PW, Casey DC, Weiss DJ, et al. Mapping Plasmodium falciparum mortality in Africa between 1990 and 2015. New England Journal of Medicine 2016;375:2435-45.

99 Ssempiira J, Nambuusi B, Kissa J, et al. Geostatistical modelling of malaria indicator survey data to assess the effects of interventions on the geographical distribution of malaria prevalence in children less than 5 years in Uganda. PLoS One 2017;12:e0174948.

100 Millar J, Psychas P, Abuaku B, et al. Detecting local risk factors for residual malaria in northern Ghana using Bayesian model averaging. Malar J 2018:17:343.

101 Giardina F, Kasasa S, Sié A, et al. Effects of vector-control interventions on changes in risk of malaria parasitaemia in subSaharan Africa: a spatial and temporal analysis. Lancet Glob Health 2014;2: e601-15.

102 Rouamba T, Samadoulougou S, Tinto H, et al. Bayesian spatiotemporal modeling of routinely collected data to assess the effect of health programs in malaria incidence during pregnancy in Burkina Faso. Sci Rep 2020;10:2618.

103 Selemani M, Msengwa AS, Mrema S, et al. Assessing the effects of mosquito nets on malaria mortality using a space time model: a case study of Rufiji and Ifakara Health and Demographic Surveillance System sites in rural Tanzania. Malar J 2016;15:257.

104 Sewe MO, Ahlm C, Rocklöv J. Remotely sensed environmental conditions and malaria mortality in three malaria endemic regions in Western Kenya. PLoS One 2016;11:e0154204.

105 Bejon P, Williams TN, Nyundo C, et al. A micro-epidemiological analysis of febrile malaria in coastal Kenya showing hotspots within hotspots. eLife 2014;3:e02130.

106 Belay DB, Kifle YG, Goshu AT, et al. Joint Bayesian modeling of time to malaria and mosquito abundance in Ethiopia. BMC Infect Dis 2017;17:415.

107 Kifle MM, Teklemariam TT, Teweldeberhan AM, et al. Malaria risk stratification and modeling the effect of rainfall on malaria incidence in Eritrea. J Environ Public Health 2019;2019:1-11.

108 Nyadanu SD, Pereira G, Nawumbeni DN, et al. Geo-visua integration of health outcomes and risk factors using excess risk and conditioned choropleth maps: a case study of malaria incidence and sociodemographic determinants in Ghana. BMC Public Health 2019;19:514.

109 Chirombo J, Ceccato P, Lowe R, et al. Childhood malaria case incidence in Malawi between 2004 and 2017: spatio-temporal modelling of climate and non-climate factors. Malar J 2020;19.

110 Nguyen M, Howes RE, Lucas TCD, et al. Mapping malaria seasonality in Madagascar using health facility data. BMC Med 2020;18:26.
111 Ouédraogo M, Kangoye DT, Samadoulougou S, et al. Malaria case fatality rate among children under five in Burkina Faso: an assessment of the spatiotemporal trends following the implementation of control programs. Int J Environ Res Public Health 2020;17:1840.

112 Simon C, Moakofhi K, Mosweunyane T, et al. Malaria control in Botswana, 2008-2012: the path towards elimination. Malar J 2013;12:458.

113 Bisanzio D, Mutuku F, LaBeaud AD, et al. Use of prospective Hospital surveillance data to define spatiotemporal heterogeneity of malaria risk in coastal Kenya. Malar J 2015;14:482.

114 Ihantamalala FA, Rakotoarimanana FMJ, Ramiadantsoa T, et al Spatial and temporal dynamics of malaria in Madagascar. Malar $J$ 2018;17:58.

115 Mukonka VM, Chanda E, Kamuliwo M, et al. Diagnostic approaches to malaria in Zambia, 2009-2014. Geospat Health 2015;10.

116 Ikeda T, Behera SK, Morioka Y, et al. Seasonally lagged effects of climatic factors on malaria incidence in South Africa. Sci Rep 2017;7:2458.

117 Kamuliwo M, Kirk KE, Chanda E, et al. Spatial patterns and determinants of malaria infection during pregnancy in Zambia. Trans R Soc Trop Med Hyg 2015;109:514-21.

118 DePina AJ, Andrade AJB, Dia AK, et al. Spatiotemporal characterisation and risk factor analysis of malaria outbreak in Cabo Verde in 2017. Trop Med Health 2019;47:3.

119 Ssempiira J, Kissa J, Nambuusi B, et al. The effect of case management and vector-control interventions on space-time patterns of malaria incidence in Uganda. Malar J 2018;17:162.

120 Mirghani SE, Nour BYM, Bushra SM, et al. The spatial-temporal clustering of Plasmodium falciparum infection over eleven years in Gezira state, the Sudan. Malar J 2010;9:172.

121 Mwakalinga VM, Sartorius BKD, Mlacha YP, et al. Spatially aggregated clusters and scattered smaller loci of elevated malaria vector density and human infection prevalence in urban Dar ES Salaam, Tanzania. Malar J 2016;15:135.

122 Shaffer JG, Touré MB, Sogoba N, et al. Clustering of asymptomatic Plasmodium falciparum infection and the effectiveness of targeted malaria control measures. Malar J 2020;19:33.

123 Bousema T, Drakeley C, Gesase S, et al. Identification of hot spots of malaria transmission for targeted malaria control. $J$ Infect Dis 2010;201:1764-74.

124 Ferrari G, Ntuku HM, Schmidlin S, et al. A malaria risk map of Kinshasa, Democratic Republic of Congo. Malar J 2016;15:27.

125 Gaudart J, Poudiougou B, Dicko A, et al. Space-Time clustering of childhood malaria at the household level: a dynamic cohort in a Mali village. BMC Public Health 2006;6:286.

126 Ishengoma DS, Mmbando BP, Mandara $\mathrm{Cl}$, et al. Trends of Plasmodium falciparum prevalence in two communities of Muheza district north-eastern Tanzania: correlation between parasite prevalence, malaria interventions and rainfall in the context of reemergence of malaria after two decades of progressively declining transmission. Malar J 2018;17:252.

127 Kangoye DT, Noor A, Midega J, et al. Malaria hotspots defined by clinical malaria, asymptomatic carriage, PCR and vector numbers in a low transmission area on the Kenyan coast. Malar $J$ 2016;15:213.

128 Kigozi R, Zinszer K, Mpimbaza A, et al. Assessing temporal associations between environmental factors and malaria morbidity at varying transmission settings in Uganda. Malar $\mathrm{J}$ 2016;15:511.

129 Ssempiira J, Kissa J, Nambuusi B, et al. Interactions between climatic changes and intervention effects on malaria spatiotemporal dynamics in Uganda. Parasite Epidemiol Control 2018;3:e00070.

130 Midekisa A, Senay G, Henebry GM, et al. Remote sensing-based time series models for malaria early warning in the highlands of Ethiopia. Malar J 2012;11:165.

131 Mabaso MLH, Craig M, Vounatsou P, et al. Towards empirical description of malaria seasonality in southern Africa: the example of Zimbabwe. Trop Med Int Health 2005;10:909-18.

132 Snow RW, Gouws E, Omumbo J, et al. Models to predict the intensity of Plasmodium falciparum transmission: applications to the burden of disease in Kenya. Trans $R$ Soc Trop Med Hyg 1998;92:601-6

133 Awine T, Malm K, Peprah NY, et al. Spatio-Temporal heterogeneity of malaria morbidity in Ghana: analysis of routine health facility data. PLoS One 2018;13:e0191707.

134 Peterson I, Borrell LN, El-Sadr W, et al. A temporal-spatial analysis of malaria transmission in Adama, Ethiopia. Am J Trop Med Hyg 2009;81:944-9. 
135 Siraj AS, Bouma MJ, Santos-Vega M, et al. Temperature and population density determine reservoir regions of seasonal persistence in highland malaria. Proc Biol Sci 2015;282:20151383.

136 Kleinschmidt I, Sharp B, Mueller I, et al. Rise in malaria incidence rates in South Africa: a small-area spatial analysis of variation in time trends. Am J Epidemiol 2002;155:257-64.
137 Seyoum D, Yewhalaw D, Duchateau L, et al. Household level spatio-temporal analysis of Plasmodium falciparum and Plasmodium vivax malaria in Ethiopia. Parasit Vectors 2017;10:196.

138 Mukonka VM, Chanda E, Haque U, et al. High burden of malaria following scale-up of control interventions in Nchelenge district, Luapula Province, Zambia. Malar J 2014;13:153. 